\title{
Editorial
}

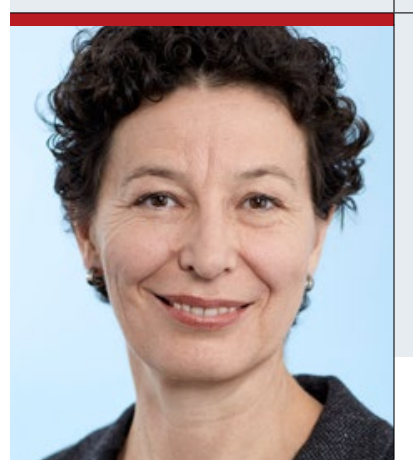

Nicoletta Eckardt

Redakteurin

nicoletta.eckardt@springer.com

facebook.com/springerpflege

\section{Raus mit der Sprache}

$\mathrm{R}$ und 80 Prozent unserer Zeit verbringen wir Studien zufolge mit Kommunikation. Doch viel bedeutet nicht zwangsläufig gut. Missverständnisse sind an der Tagesordnung, das erleben wir täglich. Manchmal können Kommunikationspannen lustig sein, oft sind sie ärgerlich. Gerade im Gesundheitswesen können Missverständnisse aber rasch gefährlich werden. Das berichtete die Schweizer Kommunikationsforscherin Prof. Annegret Hannawa auf einer Pressekonferenz zum 3. Internationalen Tag der Patientensicherheit. Anhand von WHO-Daten hat Hannawa erschreckende Zahlen errechnet: Demnach erleidet alle zwei bis sechs Sekunden ein Patient aufgrund unsicherer Kommunikation vermeidbaren Schaden. Und sie nennt die Hürden, die einer gelungenen Gesprächsführung im Wege stehen: Zeitdruck, Hierarchien, aber auch die Tatsache, dass hier Menschen mit verschiedensten Hintergründen aufeinandertreffen.

Aufgerufen zu dem Aktionstag am 17. September hatte für Deutschland das Aktionsbündnis Patientensicherheit (APS). Unter dem Motto „Wenn Schweigen gefährlich ist" machte sich das APS in diesem Jahr für eine klare Kommunikation im Gesundheitswesen stark. Dazu zählt auch eine „SpeakUp-Kultur": Sicherheitsbedenken sollen jederzeit angesprochen werden können - ohne Tabus. Das will geübt sein. So spricht laut APS nur eine von zehn Fachpersonen beobachtete Fehler oder Regelverletzungen an. Wie gefährlich Schweigen sein kann, macht auch der Beitrag von Prof. Karl H. Beine deutlich (ab S. 38).

\section{Viel Freude beim Fortbilden mit HEILBERUFE Ihre}

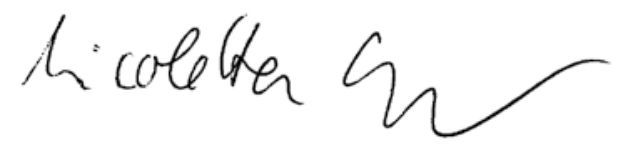

Anregungen, Wünsche, Fragen oder Lob?

Schreiben Sie uns: nicoletta.eckardt@springer.com

\section{HEILBERUFE SPEZIAL}

\section{Palliativ Care}

Was brauchen Menschen, die im Sterben liegen? Im HEILBERUFE spezial "Palliativ Care" geben namhafte Autoren Antworten auf die Frage, was Pflegende tun können, um Patienten - trotz zum Tode führender Erkrankung - die Lebensqualität durch effektive Symptomlinderung bis zuletzt zu erhalten. Sie widmen sich beispielsweise der Pflege Sterbender in der Onkologie, Geriatrie und Pädiatrie. Was muss beachtet werden, wenn Sterbende an Demenz leiden? Themen wie Trauer, Rituale, Kommunikation, Unterstützung Angehöriger stehen ebenso im Fokus wie SAPV, Patientenverfügung, Vorsorgevollmacht und Sterbehilfe.

HEILBERUFE spezial Palliative Care / 9,90 Euro / ab sofort zu bestellen:

www.springerpflege.de oder heilberufe@springer.com
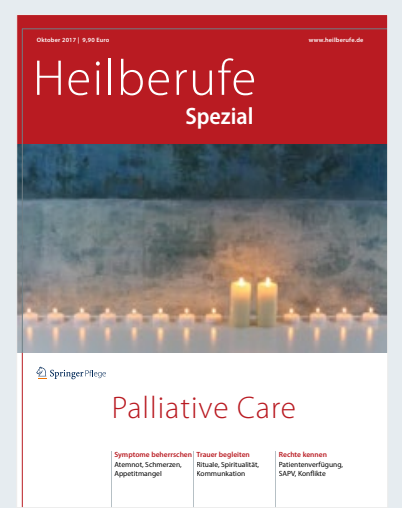